\title{
Functional Diagnosis of Hibernoma by FDG Imaging Under Propranolol Treatment: A Case Report
}

Bongioanni $\mathrm{F}^{1^{*}}$, Pomposelli $\mathrm{E}^{2}$, Pennone $\mathrm{M}^{2}$, Fiz $\mathrm{F}^{1}$, Cittadini $\mathrm{G}^{3}$, Ratto $\mathrm{GB}^{4}$, Morbelli $\mathrm{S}^{2}$, Marini $\mathrm{C}^{5}$ and Sambuceti $\mathrm{G}^{1,2}$

${ }^{1}$ Nuclear Medicine Unit, Dept of Health Sciences, University of Genoa, IRCCS San Martino- IST, Genoa, Italy

${ }^{2}$ Nuclear Medicine, IRCCS San Martino IST-Genoa, Italy

${ }^{3}$ Radiology Unit, IRCCS San Martino- IST, Genoa, Italy

${ }^{4}$ Thoracic Surgery Unit, IRCCS San Martino- IST, Genoa, Italy

${ }^{5} \mathrm{CNR}$ Institute of Bioimages and Molecular Physiology, Milan, Section of Genoa, Italy

\begin{abstract}
Positron Tomography/Computer Tomography (PET/CT) imaging of 18F-Fluorodeoxyglucose (18F-FDG) uptake is frequently used to recognize malignant nature of abnormal findings at anatomical investigations. However, several benign structures can mimic cancer glucose avidity. This case report pertains to a large mediastinal mass, incidentally detected on a coronary Computer Tomography (CT) study and showing heterogeneous signal hyperintensity in T1weighted sequences at a subsequent Magnetic Resonance Imaging (MRI). CT-guided biopsy failed to provide adequate specimen; 18F-FDG PET/CT documented an extremely high glucose analogue consumption (maximum Standardized Uptake Value: 29). In order to exclude every alternative possibility before attempting a potentially hazardous surgery, we hypothesized the possible presence of a hibernoma. To test this hypothesis, we verified the influence of sympathetic innervation on lesion uptake, by repeating 18F-FDG imaging under beta-blockade: this scan documented the complete disappearance of the anomalous tracer uptake. A 6-months follow-up scan documented stable lesion volume and persistently absent $18 \mathrm{~F}-\mathrm{FDG}$ uptake under the beta-blockers, thus presumably excluding the malignant nature of the increased tracer uptake.
\end{abstract}

Keywords: Mediastinal mass; FDG; PET/CT; Hibernoma

Abbreviations: PET/CT: Positron Tomography/Computer Tomography; FDG: ${ }^{18}$ F-Fluoro-deoxyglucose (FDG); CT: Computed Tomography; MRI: Magnetic Resonance Imaging; SUV: Standardized Uptake Value

\section{Introduction}

The high glucose consumption typical of almost all solid tumors (the so called "Warburg effect") represents the basis for PET/CT imaging of ${ }^{18} \mathrm{~F}$-Fluorodeoxy-glucose (FDG) uptake in cancer diagnosis and staging [1]. This technique became a standard in clinical oncology; nevertheless, its diagnostic accuracy is hampered by a low, yet significant, number of false positive findings. In fact, high FDG retention can be observed in benign lesion originating from tissues characterized by high glycolytic rate. This finding frequently occurs in benign neoplasms originating from brown fat, defined as "hibernomas", whose low prevalence $(1.6 \%$ of benign lipomatous tumors) often prevents a correct identification [2-4]. Actually, these lesions are most frequently observed in areas occupied by brown persists after fetal life (mediastinum, retroperitoneum, as well as paravertebral, supra-clavear and laterocervical spaces) [5]. However, connective-tissue-derived malignant neoplasms (liposarcoma or high-grade rhabdomyosarcoma) can often originate in these same sites challenging the diagnostic accuracy of FDG uptake and morphological features interrogated by MRI or X ray CT. On the other hand, even histological or cytological evaluation is often hampered by the difficult approach for fine-needle aspiration cytology or excisional biopsy. In the present report, we describe a case report in which malignant fat-derived lesion was noninvasively excluded using a pathophysiological approach.

\section{Case Report}

A 75-year-old patient was referred to our institution for the metabolic characterization of a large mass in the upper anterior mediastinum, incidentally detected during a Computer Tomography (CT) coronary angiographic study. The study had been executed due to previous detection of ascending aorta ectasia, in a patient affected by several cardio-vascular risk factors (hypertension, diabetes, history of smoking, obesity). At that time the patient was under active treatment with calcium channel blockers, aspirin and metformin but not with beta-blockers.

CT images displayed a scarcely defined mass, with heterogeneous densitometric values within the fat range that presented inhomogeneous contrast enhancement (Figure 1A). Subsequent MRI demonstrated mixed high signal intensity in T1-weighted sequences, mild hyperintensity in $\mathrm{T} 2$, and a heterogeneous signal loss in fatsuppressed acquisitions. The lesion displayed only mild mass effect on the surrounding structures (Figure 1B-C). CT-guided biopsy was then attempted but did not provided adequate specimen [6].

In this context, presence of malignancy couldn't be excluded and the patient was referred to FDG PET/CT scan. Image acquisition was performed 50 minutes after administration of $5 \mathrm{MBq}$ of $18 \mathrm{~F}-\mathrm{FDG}$ per $\mathrm{Kg}$ of patient weight. At tracer injection, the patient was fasting since 12 hours and lying in a recumbent position in a quiet room with an adequate temperature. Scan was performed using a 3D whole-body PET scan and a non-diagnostic CT scan protocol, for attenuation correction and anatomical localization. These parameters were used in all subsequent studies. The study demonstrated an extremely high tracer uptake within the mass (maximum SUV of 29), without any other

*Corresponding author: Francesca Bongioanni, Nuclear Medicine Unit, Dept of Health Sciences, University of Genoa, IRCCS San Martino- IST, Genoa, Italy, Tel: 3901020991; E-mail: bongioannifrancesca@virgilio.it

Received April 23, 2015; Accepted June 06, 2015; Published June 13, 2015

Citation: Bongioanni F, Pomposelli E, Pennone M, Fiz F, Cittadini G, et al. (2015) Functional Diagnosis of Hibernoma by FDG Imaging Under Propranolol Treatment: A Case Report. J Health Med Informat 6: 190. doi:10.4172/2157-7420.1000190

Copyright: ( 2015 Bongioanni F. This is an open-access article distributed under the terms of the Creative Commons Attribution License, which permits unrestricted use, distribution, and reproduction in any medium, provided the original author and source are credited. 
Citation: Bongioanni F, Pomposelli E, Pennone M, Fiz F, Cittadini G, et al. (2015) Functional Diagnosis of Hibernoma by FDG Imaging Under Propranolol Treatment: A Case Report. J Health Med Informat 6: 190. doi:10.4172/2157-7420.1000190
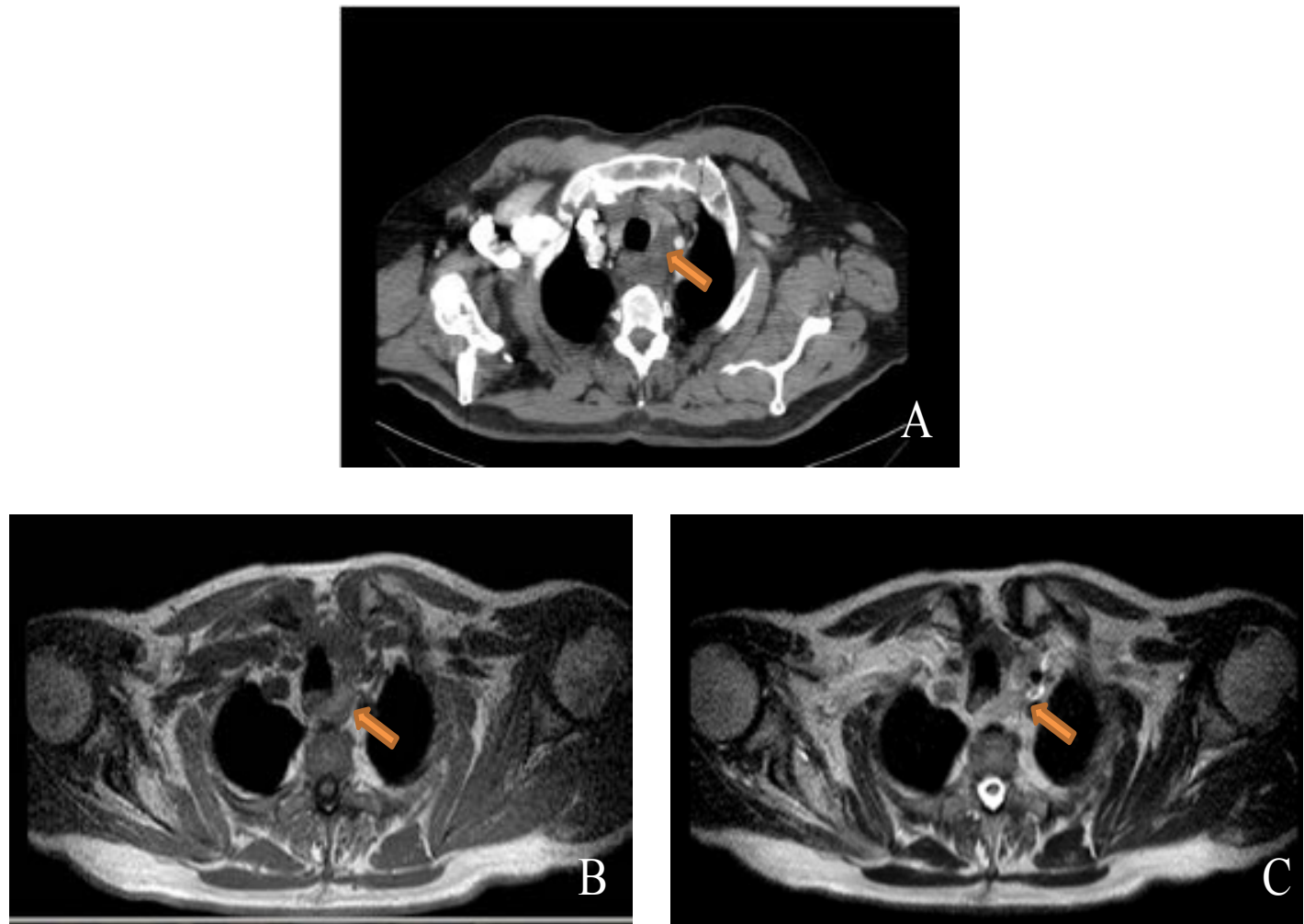

Figure 1: CT scanning and MRI study show a mediastinal mass.

A: CT coronary angiographic study highlights a lesion with distribution of densitometric values prevalently located within the fat range. This mass, located on the left paratracheal groove, shows a highly heterogeneous contrast enhancement pattern.

Magnetic resonance imaging demonstrate a mixed high signal intensity in T1-weighted sequences (B): Mild hyperintensity in T2

(C) With a heterogeneous signal loss in fat suppressed acquisition sequences.

anomalous findings (Figure 2A). This finding suggested a malignant nature of the lesion. Nevertheless, anatomical site did not permit to exclude a hibernoma mostly because the scan was performed during an extremely cold winter, possibly triggering adrenergic stimulation of brown fat glucose consumption.

Accordingly, lesion nature remained undefined while its surgical excision entailed a considerable procedural risk, owing to mass size and relationships with both esophagus and left carotid artery. To justify the hazard related to surgery we thus planned to characterize the pathophysiological mechanisms underlying the high FDG uptake. To this purpose we planned to verify whether the intense glucose consumption was related to adrenergic activation by repeating PET/CT scanning under full dose beta-blocker therapy [7-9]. Repeated study, under propranolol treatment $(120 \mathrm{mg}$ per day, starting 5 days before the exam) documented the virtual disappearance of FDG uptake within the lesion, with a SUV max of 1.1 (Figure 2B).

This finding was interpreted as a marker of a benign lesion nature and indicated a strategy shift from the possible surgical option to a close follow up program that implied to repeat PET/CT scan after 6 months. The study was performed under the same propranolol treatment regimen and confirmed the absence of FDG uptake (SUV max 0.9 ) within the lesion that moreover, displayed the same volume at coregistered CT images (Figure 2C).

\section{Discussion}

Hibernoma is a rare asymptomatic and benign lesion. Its diagnostic definition is hampered by the lack of morphologic criteria able to accurately differentiate this rare incidental from other malignant lesions such as liposarcoma, rhabdomyosarcoma, giant cell tumor and lymphoma [6,10-12]. A similar misdiagnosis can occur at PET/CT imaging since the high FDG intake can reflect either a Warburg effect of a rapidly proliferating high-grade mesenchimal tumors and a high glycolytic flux caused by adrenergic activation of brown fat to preserve body temperature under cold exposure. Accordingly, this differential diagnosis most often asks for cytological or histological evaluation $[13,14]$.

Actually, brown fat behavior reflects the integrated response against intense cold, which is mediated by the adrenergic system and comprises vasoconstriction (Alpha-1 receptors), shivering (Beta2 receptors) and, especially in infants and young adults, stimulation of heat production by the brown adipose tissue (Beta- 3 receptors). Hibernomas are benign, well differentiated lesions, and maintain the functional characteristics of the original tissue. In patients submitted to PET/CT in winter, the accelerated glucose metabolism can simulate malignant lesions at FDG PET/CT imaging [14]. Yet, the physiological regulation of this metabolic pattern, and thus the benign nature of the tissue can be documented by pharmacologic interventions able to reduce tracer retention in physiological brown tissue [7-9].

This case presents an unusual sequence of diagnostic test planning in which the functional study permitted to define an otherwise uncertain diagnosis. The complete first-line workup strongly suggested a malignant lesion and the need for a potentially hazardous surgical 
Citation: Bongioanni F, Pomposelli E, Pennone M, Fiz F, Cittadini G, et al. (2015) Functional Diagnosis of Hibernoma by FDG Imaging Under Propranolol Treatment: A Case Report. J Health Med Informat 6: 190. doi:10.4172/2157-7420.1000190
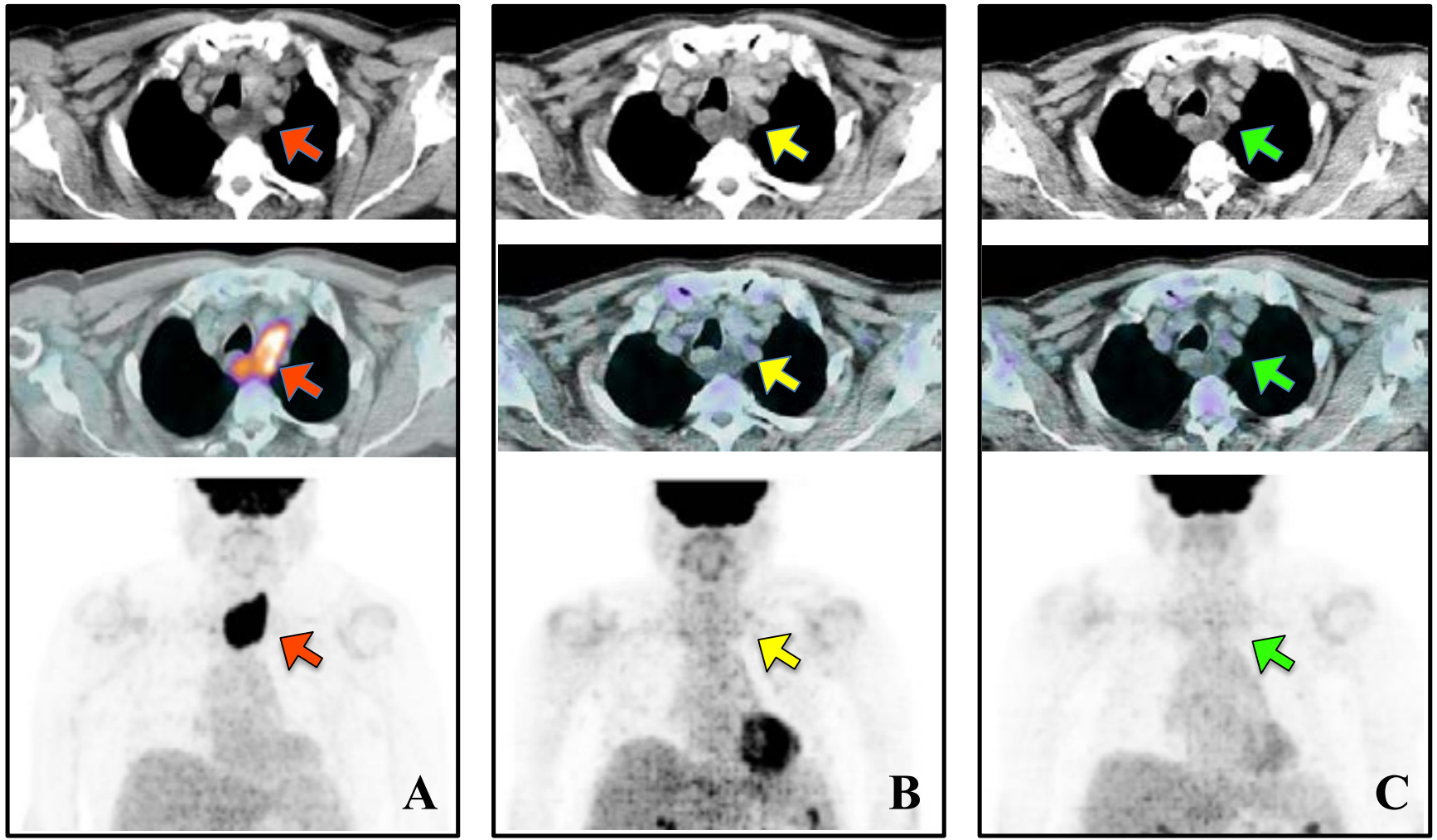

Figure 2: PET/CT scans: baseline imaging and after treatment with beta-blockers.

A: Baseline 18F-FDG PET/CT scan shows an extremely high FDG uptake within the mediastinal mass (SUV max 29) suspicious for hypermetabolic malignant lesion.

B: PET/CT scan after five days of treatment with propranolol $(120 \mathrm{mg} /$ die $)$ demonstrate a complete disappearance of tracer hyperaccumulation within the mass. C: PET/CT scan after six months, under the same treatment protocol, confirmed an absent FDG uptake within the lesion.

approach. The only non-invasive way to rule out this scenario was to consider the possible pharmacologic modulation of metabolic signal. To this purpose, we selected the unselective propranolol whose action on beta- 3 receptors has been already documented [15,16], although the effectiveness of other beta-blockers was not tested. The inclusion of this possibility in the differential diagnosis and planning functional imaging procedures was, in this case, the key for this diagnosis.

There are of course several limitations intrinsic to this approach. First, the CT scanning protocol that is used in hybrid PET/CT imaging is "low dose", for the sake of limiting the patients' exposure to ionizing radiations, therefore limiting our anatomic definition of the lesion and its changes in follow-up scans. However, the complete metabolic response after beta-blockade, the absence of macroscopic modifications and the lack of local invasion or distant localizations at follow-up scans strongly support the hypothesis of a benign lesion $[17,18]$.

Moreover, no further biopsies were attempted, given the inconclusive result of the first one. An excisional biopsy might theoretically have clarified the diagnosis, but the risk/benefit ratio was too high (also considering the negative follow-up imaging) to justify such an approach.

This report confirms the need of considering, planning and carefully interpreting functional imaging and decoding its meaning on the basis of the underlying pathophysiological mechanisms.

\section{Consent}

Written informed consent was obtained from the patient for publication of this Case Report and any accompanying images. A copy of the written consent is available for review by the Editor of this journal.

\section{Acknowledgements}

We thank Professor Lorenzo Derchi from University of Genoa IRCCS-San Martino -IST who provided precious advices on interpretation of radiological images.

\section{Competing Interest}

This study was not financed by any science foundation or support program The authors have no competing interest, including relevant financial interest activities and affiliations.

\section{References}

1. Pauwels EK, Ribeiro MJ, Stoot JH, McCready VR, Bourguignon M, et al. (1998) FDG accumulation and tumor biology. Nucl Med Biol 25: 317-322.

2. Ahn C, Harvey JC (1990) Mediastinal hibernoma, a rare tumor. Ann Thorac Surg 50: 828-830.

3. Udwadia ZF, Kumar N, Bhaduri AS (1999) Mediastinal hibernoma. Eur J Cardiothorac Surg 15: 533-535

4. Anderson SE, Schwab C, Stauffer E, Banic A, Steinbach LS (2001) Hibernoma: imaging characteristics of a rare benign soft tissue tumor. Skeletal Radiol 30 590-595.

5. Heaton JM (1972) The distribution of brown adipose tissue in the human. J Anat 112: 35-39. 
Citation: Bongioanni F, Pomposelli E, Pennone M, Fiz F, Cittadini G, et al. (2015) Functional Diagnosis of Hibernoma by FDG Imaging Under Propranolol Treatment: A Case Report. J Health Med Informat 6: 190. doi:10.4172/2157-7420.1000190

6. Hertoghs M, Van Schil P, Rutsaert R, Van Marck E, Vallaeys J (2009) Intrathoracic hibernoma: report of two cases. Lung Cancer 64: 367-370.

7. Gelfand MJ, O'hara SM, Curtwright LA, Maclean JR (2005) Pre-medication to block [(18)F]FDG uptake in the brown adipose tissue of pediatric and adolescent patients. Pediatr Radiol 35: 984-990.

8. Söderlund V, Larsson SA, Jacobsson H (2007) Reduction of FDG uptake in brown adipose tissue in clinical patients by a single dose of propranolol. Eur $\mathrm{J}$ Nucl Med Mol Imaging 34: 1018-1022.

9. Parysow O, Mollerach AM, Jager V, Racioppi S, San Roman J, et al. (2007) Low-dose oral propranolol could reduce brown adipose tissue F-18 FDG uptake in patients undergoing PET scans. Clin Nucl Med 32: 351-357.

10. Ong SY, Maziak DE, Shamji FM, Matzinger FR, Perkins DG (2002) Intrathoracic hibernoma. Can J Surg 45: 145-146.

11. Kransdorf MJ, Bancroft LW, Peterson JJ, Murphey MD, Foster WC, et al. (2002) Imaging of fatty tumors: distinction of lipoma and well-differentiated liposarcoma. Radiology 224: 99-104.
12. da Motta AC, Tunkel DE, Westra WH, Yousem DM (2006) Imaging findings of a hibernoma of the neck. SeeAJNR Am J Neuroradiol 27: 1658-1659.

13. Lin E, Alavi A (2005) PET and PET/CT: a clinical guide. Thieme medical pulisher: inc 32-35

14. Subramaniam RM, Clayton AC, Karantanis D, Collins DA (2007) Hibernoma: 18F FDG PET/CT imaging. J Thorac Oncol 2: 569-570.

15. Wu MJ, Shin DH, Kim MY, Park CG, Kim YD, et al. (2015) Functional effects of $\beta 3$-adrenoceptor on pacemaker activity in interstitial cells of Cajal from the mouse colon. Eur J Pharmacol 754: 32-40.

16. van Wieringen JP, Michel-Reher MB, Hatanaka T, Ueshima K, Michel MC (2013) The new radioligand [(3)H]-L 748,337 differentially labels human and rat ß3-adrenoceptors. Eur J Pharmacol 720: 124-130.

17. Baldi A, Santini M, Mellone P, Esposito V, Groeger AM, et al. (2004) Mediastinal hibernoma: a case report. J Clin Pathol 57: 993-994.

18. Mavrogenis AF, Coll-Mesa L (2012) Soft tissue: Hibernoma. Atlas Genet Cytogenet Oncol Haematol. 